\title{
Dynamic Cooperation in Local Public Goods Supply with Imperfect Monitoring
}

\section{Guillaume Cheikbossian}

Université Montpellier 1

Toulouse School of Economics (GREMAQ)

\section{Wilfried Sand-Zantman}

Toulouse School of Economics (IDEI and GREMAQ)

Université de Toulouse

January, 2010

\footnotetext{
${ }^{\star}$ E-mail: cheikbossian: guillaume.cheikbossian@univ-tlse1.fr; sand-zantman: wsandz@cict.fr

We thank Patrick Gonzalez, Johannes Hörner and seminar participants at the Université de Montréal, Université Laval (Québec), Canadian Economic Association 2006 and European Economic Association 2006 (Vienna) annual conferences for comments and suggestions. The usual disclaimer applies.
} 


\begin{abstract}
This paper develops a two-country model where each invests in a local public good which produces positive externalities. Each country's effort investment is private information and cannot be directly observed. It is only inferred from the observed level of public output which depends on the investment effort devoted towards its production. In a repeated game setting, we characterize the condition for the existence of a cut-off trigger strategy equilibrium such that the two countries have no incentives to deviate from full cooperation. If it exists, this cut-off point is shown to be decreasing in the spillover parameter and in the length of the punishment period. Finally, we show that increasing the correlation between the two country-specific shocks surprisingly restricts the prospects for cooperation.
\end{abstract}

Keywords: Local Public Goods, Externality, Imperfect Information, Repeated Game.

JEL Classification: H7, C73 


\section{Introduction}

In the presence of spillovers, decentralized provision of local public goods leads to an inefficient outcome. If, however, local jurisdictions have repeated interactions, they may tacitly cooperate so as to internalize cross-border externalities. Indeed, according to the "folk theorems" cooperation can be sustained as a Nash equilibrium of a repeated game by strategies of reciprocity as long as one's partner does not discount the future too heavily. Our objective in this paper is then to explore the consequences of imperfect information with respect to the cost levels of the local public goods, provided by neighboring jurisdictions, on the sustainability of efficient outcomes in an infinitely repeated game.

The literature on local public good provision typically deals with perfect information situations. In several circumstances, however, there is an information asymmetry with respect to local cost conditions. One can think of the costs of research and development activities or the costs of investment in environmental quality. For example, Cornes and Silva (2000, 2002) refer to an analysis of Mäler (1991) who noted about the Acid rain problem in Europe that "the control costs and environmental damage in one particular country is known to that country only". It is also well recognized in the literature on fiscal federalism that private information with respect to both preferences and cost conditions raises important challenges for the design of transfer schemes at the central level (e.g., Costello (1993)). Clearly, imperfect information about technology and local cost conditions may impose significant constraints on the ability of local jurisdictions to achieve an efficient outcome. In particular, in a dynamic setting, this can decrease the effectiveness of the threat of non-cooperation in the future (to sustain cooperation today) since deviations from cooperation may only imperfectly detected.

We develop a two-country model where each provides a local public good. These public goods produce spillover benefits which are enjoyed by residents of the other country. The output of the public good in each country is a function of the cost of effort devoted towards its production plus a country-specific shock. While public output in each country is perfectly observed, the cost of effort in each country is private information and thereby cannot be directly observed in the other country. Finally, we consider that the situation is repeated over time so that the two countries may tacitly cooperate and internalize externalities through decentralized strategies of reciprocity. Specifically, we focus in this paper on cut-off trigger strategy equilibria. In other words, each country produces the optimal level of effort as long as each country's realized public output is above a given level. If realized public output in one or both country falls below this level or cut-off point, the two 
countries switch to the static non-cooperative outcome for $T$ periods regardless of what happens to public output in each country during this period. At the end of the non-cooperative episode, i.e. $T+1$ periods after public output in one or both countries had fallen, they will resume cooperation.

We characterize a sufficient condition for the existence of such a cut-off point (also called the trigger public output) such that cooperation can be sustained. We then show that this cut-off point decreases both in the length of the punishment period and in the spillover parameter. In other words, as the spillover effect or the number of periods of punishment increases, it becomes more likely that the two countries maintain cooperation. We analyze, in turn, the impact of the correlation between the two country-specific shocks on the incentives for each country to produce the optimal level of effort. Interestingly, we show that increasing the correlation coefficient from 0 reduces the prospects for cooperation in the sense that it decreases, for a given level of the cut-off point, the marginal benefit of exerting effort compared to the case of independent shocks.

The present paper is related to the general problem of tacit cooperation in a dynamic game setting with imperfect monitoring initiated by Porter (1983) and Green and Porter (1984). Although, this problem has been extensively analyzed in dynamic oligopoly models, it has not been explored in a model of provision of local public goods. For example, McMillan (1979) and more recently Pecorino (1999) analyze a repeated game setting for the private provision of public good but without uncertainty. Recently, public economists have put under scrutiny the implications of imperfect information on the costs of providing local public services, in particular in a static setting, for the allocation of resources between members of a federation (see, e.g. Lockwood (1999) and references therein). In the present paper, we analyze the implications of information asymmetry with respect to cost levels of local public goods on the ability of independent jurisdictions to sustain cooperation in a repeated game setting.

Our analysis is also related to the literature on inter-governmental yardstick competition initiated by Salmon (1987) and Besley and Case (1995). ${ }^{1}$ Indeed, when there is shock-interdependence the evaluation of government policy in one country depends on government performance in neighboring countries. In a static setting, yardstick competition mechanisms that rely on an informational externality in general help to enhance efficiency. In a dynamic setting with no monetary transfers between parties, we show that such a mechanism may restrict the prospects for future cooperation.

The paper is organized as follows. We begin in Section 2 by presenting the model. In Section

\footnotetext{
${ }^{1}$ This theory has been further developed by, among others, Sand-Zantman (2004), Belleflamme and Hindriks (2005), Revelli (2006), and Besley and Smart (2007).
} 
3, we characterize the cut-off trigger strategy equilibrium of the repeated game with imperfect monitoring. In Section 4, we examine how shock-interdependence affects this equilibrium outcome. Section 5 offers a brief conclusion.

\section{The Stage Game}

We consider a world consisting of two neighboring countries. Each country has a population size normalized to 1 and there is no mobility across countries. In each country all individuals have identical endowments $y$ and consume a private good and two public services, each one associated with a particular country. A given level of investment $e_{j}$ in the $j$ th country will give the following level of public services in that country

$$
q_{j}=e_{j}+\varepsilon_{j} \quad j=1,2
$$

where $e_{j}$ is the level of investment or effort (number of civil servants, level of infrastructure) chosen by the government of the $j$ th country. $e_{j}$ is not observable by the citizens of country $k . \varepsilon_{j}$ is a random shock that follows a normal law with mean 0 and variance $\sigma^{2}$ for $j=1,2$. Individual private consumption in country $j$ is $x_{j}=y-c\left(e_{j}\right)$ where $c\left(e_{j}\right)$ is the cost of producing $e_{j}$ units of effort investments in public goods. We assume a convex cost function as it follows

$$
c\left(e_{j}\right)=e_{j}^{2} / 2, \quad j=1,2 .
$$

Individuals in the two countries have the same preferences for private and public consumption. These preferences are represented by a linear utility function

$$
U_{j}=x_{j}+\frac{\left[q_{j}+\beta q_{k}\right]}{1+\beta}, \quad j \neq k
$$

where $\beta$ represents the intensity of cross-country spillovers related to public service provision. When $\beta=0$, citizens care only about the public good in their own country, while when $\beta=1$ they care equally about public spending in both countries. We also assume that the exogenous income $y$ is sufficiently high to always allow positive consumption of the private good. This implies together with linearity of preferences that there are no wealth effects.

Let $W_{j}$ be the expected level of public goods surplus in country $j$. We have $W_{j}=E\left[\frac{\left[q_{j}+\beta q_{k}\right]}{1+\beta}\right]-$ $e_{j}^{2} / 2=\frac{\left[e_{j}+\beta e_{k}\right]}{1+\beta}-e_{j}^{2} / 2$ since $\varepsilon_{j}$ has a 0 mean for $j=1,2$. Suppose first, that each country maximizes its own expected surplus $W_{j}$ with respect to $e_{j}$ given the other country's choice of effort investment. In the Nash equilibrium, both countries invest $\tilde{e}=1 /(1+\beta)$. This gives the following equilibrium 
level of expected public services surplus for both countries $W^{N}=(1+2 \beta) /\left[2(1+\beta)^{2}\right]$ which is decreasing in the spillover parameter.

If, however, both countries manage to cooperate, they maximize the sum of the expected surplus i.e. $W_{1}+W_{2}$ with respect to both $e_{1}$ and $e_{2}$. The Bowen-Lindhal-Samuelson condition gives the common optimal level of effort investment i.e. $e^{*}=1$. The level of expected public goods surplus, in that case, is then $W^{C}=1 / 2$ which is independent of the size of the spillover effect. This comes from the symmetry of the model and from our specification of the utility function that is normalized with the spillover parameter.

If each country's effort were publicly observable, it would be straightforward to show that the efficient outcome can be supported as a trigger strategy equilibrium as long as both countries are sufficiently patient. Specifically, if one country (let say country 1) defects from the cooperative outcome, it would choose the same level of effort investment as in the Nash outcome i.e. $\tilde{e}=$ $1 /(1+\beta)$. Hence, the equilibrium level of surplus of the country that defects would be $W^{D}=$

$[1+2 \beta(1+\beta)] /\left[2(1+\beta)^{2}\right]$ which is increasing in the spillover parameter. Let $v_{i}=\sum_{t=0}^{\infty} \delta^{t} W_{i}$ be the discounted sum of country $i$ 's stage game payoffs where $0<\delta<1$ is the discount factor. Then the optimal outcome can be sustained through the threat of permanently reverting to the non-cooperative outcome if and only if $\delta \geq 1 / 2$. Note that, in the case of perfect information, an infinite punishment period maximizes the prospects for cooperation because deviations are perfectly detected.

\section{Cooperation Under Imperfect Monitoring}

Consider now the repeated game with imperfect monitoring. The two countries meet each period to play the stage game described above, where each country has the objective of maximizing its expected discounted stream of public good surplus. When entering a period, a country observes only the history of its own level of effort and realized public output in the two countries. Following Green and Porter (1984) and Fudenberg, Levine, and Maskin (1994), we restrict attention to those equilibria in which countries' strategies only depend on realized public outputs and not on their own private history of policy schedule. Such strategies are called public strategies and such equilibria are called perfect public equilibria (PPE).

As is typical in repeated games, there can be many perfect public equilibria in our game many of which can involve complicated strategies. We then make two restrictions. First, as in Green and Porter (1984), we consider equilibria with two levels of effort in public investment and with symmetric strategies. In addition, we constraint the two countries to choosing either the non- 
cooperative level of effort, i.e. $\tilde{e}=1 /(1+\beta)$, or the first-best level of effort of the stage game, i.e. $e^{*}=1$. We then presuppose, as in Green and Porter (1984), special forms for the cooperative and punishment phases. More precisely, as a part of their strategies, the two countries must decide when to produce the cooperative or the non-cooperative level of effort as a function of public histories.

We also consider, for the moment, that the two country-specific shocks are independently distributed over time and across countries. Hence, high public output realizations in a particular country would tend to suggest that this country has produced the optimal level of effort while low realizations would tend to suggest that this country has defected. Abreu, Pearce and Stacchetti $(1986,1990)$ show that if the conditional distribution of the public signal given effort satisfies the Monotone Likelihood Ratio Property (MLRP) then a tail test is the optimal statistical criterion for the players to adopt. ${ }^{2}$ This implies the existence of a critical level of the observable public output denoted $\widehat{q}$ such that if public output falls below this value in any country, punishment is triggered. We finally assume that the punishment lasts for $T$ periods.

Each country then uses the following cut-off strategy : (i) to choose the optimal level of effort $e^{*}$ in the first period and to continue to do so as long as the observed level of public output in each country is as high as $\widehat{q}$ in both countries; (ii) if public output in one or both countries fall below $\widehat{q}$ at some period $t$, then to produce the non-cooperative level of effort $\widetilde{e}$ for $T$ periods and then return to the cooperative level of effort until public output in one or both countries fall (again) below $\widehat{q}$. The probability of maintaining cooperation next period is then given by

$$
\mu\left(e_{1}, e_{2}\right)=\operatorname{Prob}\left[\varepsilon_{1} \geq \widehat{q}-e_{1}\right] \cdot \operatorname{Prob}\left[\varepsilon_{2} \geq \widehat{q}-e_{2}\right] .
$$

Using the properties of the normal distribution and denoting $\Phi$ the cumulative of a standard normal law (with mean equal to 0 and variance equal to 1 ), this probability can be written as it follows

$$
\mu\left(e_{1}, e_{2}\right)=\left(1-\Phi\left[\frac{\widehat{q}-e_{1}}{\sigma}\right]\right) \cdot\left(1-\Phi\left[\frac{\widehat{q}-e_{2}}{\sigma}\right]\right) .
$$

Let $V_{t}^{C}$ (respectively $V_{t}^{P}$ ) be the present discounted value of a country's payoff from date $t$ on, assuming that at date $t$ the game is in the cooperative (respectively punishment) phase. We have

$$
V_{t}^{C}=W\left(e_{1}, e_{2}\right)+\delta\left[\mu\left(e_{1}, e_{2}\right) V_{t+1}^{C}+\left(1-\mu\left(e_{1}, e_{2}\right)\right) V_{t+1}^{P}\right]
$$

$V_{t}^{C}$ equals present period expected payoff plus expected future payoffs in present discounted value. Next period, either cooperation is continued (with probability $\mu\left(e_{1}, e_{2}\right)$ ) or the implicit contrac-

\footnotetext{
${ }^{2}$ Formally, let $F(q \mid e)$ the cumulative distribution of public output given effort, with density $f(q \mid e)$. It satisfies the MLRP if for two values of effort $e_{1}$ and $e_{2}$ with $e_{1}>e_{2}$, we have that $f\left(q \mid e_{1}\right) / f\left(q \mid e_{2}\right)$ is increasing in $q$.
} 
tual agreement is broken (with probability $1-\mu\left(e_{1}, e_{2}\right)$ ) in which case both countries enter the punishment phase and do not cooperate for $T$ periods.

We can now analyze the optimal behavior of each country. Let first state the following Lemma. ${ }^{3}$

Lemma 1 Let assume that region 2 acts cooperatively i.e. $e_{2}=e^{*}$. A necessary and sufficient condition for $e_{1}=e^{*}$ to be an individual optimal strategy for region 1 is given by the first-order condition, i.e., $\left.\frac{\partial V_{t}^{c}}{\partial e_{1}}\right|_{e_{1}=e^{*}}=0$.

Using (6), we have

$$
\left.\frac{\partial V_{t}^{C}}{\partial e_{1}}\right|_{e_{1}=e^{*}}=\left.\frac{\partial W\left(e_{1}, e^{*}\right)}{\partial e_{1}}\right|_{e_{1}=e^{*}}+\left.\delta\left[V_{t+1}^{C}-V_{t+1}^{P}\right] \frac{\partial \mu\left(e_{1}, e^{*}\right)}{\partial e_{1}}\right|_{e_{1}=e^{*}}=0 .
$$

The time invariant nature of our framework implies that if the cooperative level of effort is an optimal strategy for country 1 today, it will also be an optimal strategy for that country in the future. Hence, this condition may be written equivalently as

$$
\left.\frac{\partial V^{C}}{\partial e_{1}}\right|_{e_{1}=e^{*}}=0 \Leftrightarrow \frac{\beta}{1+\beta}-\left.\delta\left[V^{C}-V^{P}\right] \frac{\partial \mu\left(e_{1}, e^{*}\right)}{\partial e_{1}}\right|_{e_{1}=e^{*}}=0 .
$$

The first term of the above expression represents the expected marginal benefit from underproducing effort investments in public goods. When country 1 decreases its effort below the optimal level of effort, it free-rides onto the other country and the expected marginal benefit of deviation is increasing in the spillover parameter. The second term corresponds to the expected marginal loss in future payoffs from possibly triggering a punishment phase. This expected marginal cost of deviation is the product of two terms. The first term corresponds to the difference between the expected discounted payoff of cooperation and the expected discounted payoff of non-cooperation. The second term corresponds to the marginal probability that the game remains in the cooperative phase. As shown below, when country 1 decreases its effort below the optimal level of effort, it contributes to decrease the marginal probability of remaining in the cooperative phase. Therefore, the optimal level of effort $e^{*}=1$ is a best-response to the predicted action of country 2 when the expected marginal benefit exactly balance the marginal cost from under-producing public investments.

Let first characterize the difference between the expected discounted payoff of cooperation and that of non-cooperation, i.e. $V^{C}-V^{P}$. $V^{P}$ consists of playing the Nash-equilibrium for $T$ periods

\footnotetext{
${ }^{3}$ All proofs are given in the Appendix
} 
and resume cooperation at the $T+1$ th period. Formally, we have

$$
\begin{aligned}
V^{P} & =W^{N}+\delta W^{N}+\ldots+\delta^{T-1} W^{N}+\delta^{T} V^{C} \\
& =\frac{1-\delta^{T}}{1-\delta} W^{N}+\delta^{T} V^{C}
\end{aligned}
$$

Inserting this expression into equation (6) and dropping the time subscript leads to

$$
V^{C}=\frac{W^{N}}{1-\delta}+\frac{W^{C}-W^{N}}{1-\delta \mu\left(e^{*}\right)-\delta^{T+1}\left(1-\mu\left(e^{*}\right)\right)}
$$

where $\mu\left(e^{*}\right) \equiv \mu\left(e^{*}, e^{*}\right)$. Hence, using (9), we have

$$
V^{C}-V^{P}=\frac{\left(1-\delta^{T}\right)\left[W^{C}-W^{N}\right]}{1-\delta \mu\left(e^{*}\right)-\delta^{T+1}\left(1-\mu\left(e^{*}\right)\right)}
$$

Recalling that $W^{N}=[1+2 \beta] /\left[2(1+\beta)^{2}\right]$ and $W^{C}=1 / 2$. Using these expressions, the necessary and sufficient first-order condition given by (8) can then be rewritten as

$$
\frac{-\beta}{1+\beta}+\frac{\delta\left(1-\delta^{T}\right) \beta^{2}}{2(1+\beta)^{2}} \frac{\left.\frac{\partial \mu\left(e_{1}, e^{*}\right)}{\partial e_{1}}\right|_{e_{1}=e^{*}}}{1-\delta \mu\left(e^{*}\right)-\delta^{T+1}\left(1-\mu\left(e^{*}\right)\right)}=0
$$

This equilibrium condition imposes some restrictions on the level of the trigger output $\widehat{q}$ and on the structural parameters $\delta, \beta$ and $\sigma$.

Let first characterize the marginal probability that the game remains in the cooperative phase. Using (5), it is easy to see that

$$
\left.\frac{\partial \mu\left(e_{1}, e^{*}\right)}{\partial e_{1}}\right|_{e_{1}=e^{*}}=\frac{e^{-\frac{\left(\widehat{q}-e^{*}\right)^{2}}{2 \sigma^{2}}}}{\sigma \sqrt{2 \pi}} \cdot\left(1-\Phi\left[\frac{\widehat{q}-e^{*}}{\sigma}\right]\right)>0
$$

Let $G=\widehat{q}-e^{*}$ be the difference between the trigger output and the optimal level of effort. Then using (5) and (13), (12) can then be rewritten as $R(G)=0$ with

$$
R(G)=\frac{-\beta}{1+\beta}+\frac{\delta\left(1-\delta^{T}\right) \beta^{2}}{2(1+\beta)^{2}} \frac{\Phi_{G}(1-\Phi)}{1-\delta(1-\Phi)^{2}-\delta^{T+1}\left(1-(1-\Phi)^{2}\right)}
$$

where $\Phi_{G}$ is the density function and where the arguments of both $\Phi$ and $\Phi_{G}$ is $G / \sigma$. Again, the expected marginal return to a country from decreasing its effort balances exactly the marginal increase in risk of incurring a loss in returns by triggering a reversion to the non-cooperative outcome.

The following Proposition characterizes the existence of a cut-off trigger strategy equilibrium.

Proposition 1 : The $R(G)$ function given by (14) is single-peaked with a unique maximum denoted $\tilde{G}$. Hence, there exists a cut-off trigger strategy equilibrium given by $(\hat{q}, T)$ if and only if $R(\tilde{G}) \geq 0$, and where $\hat{q}$ is such that $R\left(\hat{q}-e^{*}\right)=0$. 
The $R(G)$ function has the shape as shown in figure 1. (14) has then either no solutions or two solutions, depending on whether $R(\tilde{G})$ is negative or positive.

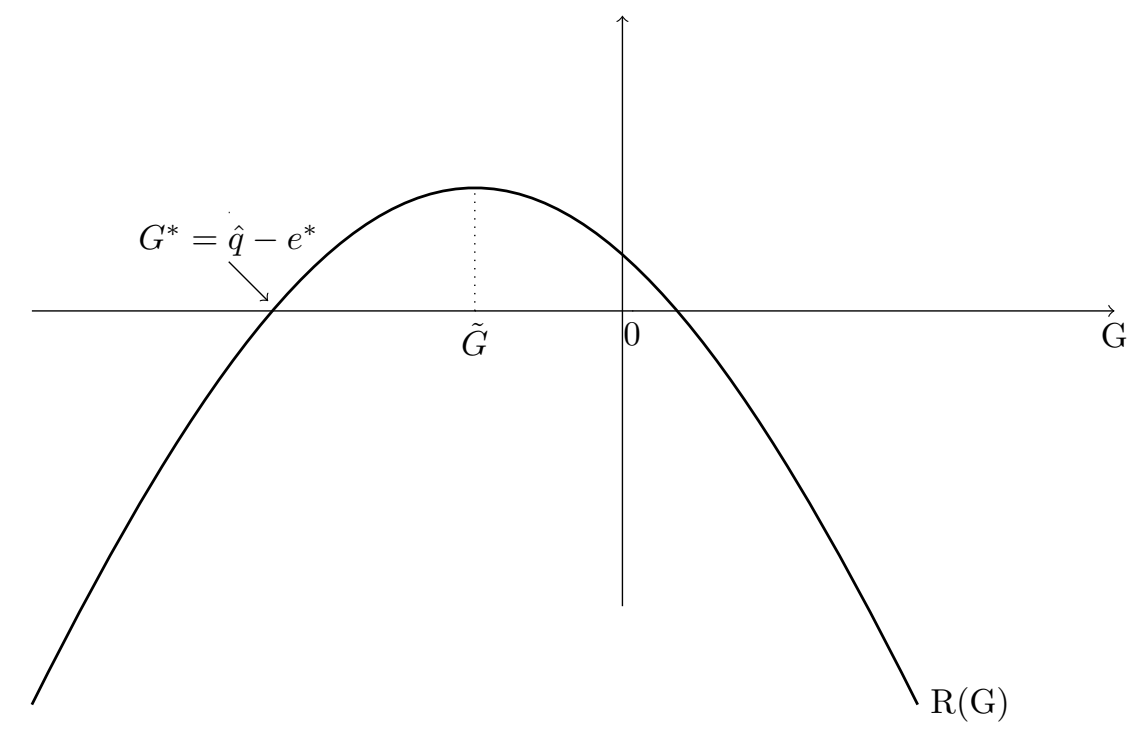

Figure 1

When $R(\tilde{G}) \geq 0, R(G)=0$ has two solutions and the smaller solution yields the cut-off point $\hat{q}^{4}$ as a function (among other things) of the level of effort $e^{*}$ that both countries produce in cooperative periods, and of $T$ which is the length of episodes during which countries revert to the static non-cooperative outcome. This proposition states that, even though there is imperfect monitoring, the first-best level of effort $e^{*}$ of the stage game can be sustained by the players' threats to revert for $T$ periods to the one-shot non-cooperative equilibrium in case of a deviation from the prescribed strategy. However, compared to the case of perfect monitoring, tacit cooperation works less well since the two countries switch (periodically) to the non-cooperative outcome on the equilibrium path. Put another way, in equilibrium, punishment is not triggered by the inference that one country deviated in the previous period. Rather, each country correctly presumes that its partner produced the optimal level of effort and that public service provision was low because of a negative shock. Reversion to the non-cooperative outcome is however necessary in this case because if the punishment did not occur when public output was low, the two countries would not have any incentives to cooperate.

It is also worth pointing out that reversionary episodes must last for some periods. Indeed, when $T=0$, we have $R(G)=-\beta /(1+\beta)<0$ which implies there does not exist a trigger strategy

\footnotetext{
${ }^{4}$ The two solutions have the same impact on efforts but the lower $G^{*}$ (hence the lower $\widehat{q}$ ), the larger is the probability that the game remains in the cooperative phase.
} 
equilibrium that supports the first-best level of effort $e^{*}$. This is also the case when the variance of each country-specific shock is large or when countries are not patient enough. To see this point, assume that the length of the punishment period is infinite, i.e. $T \rightarrow \infty$. Then the inequality $R(\tilde{G}) \geq 0$ reduces to $\delta \geq \tilde{\delta}$ with

$$
\tilde{\delta}=\frac{2(1+\beta)}{(1-\Phi)\left[\beta \Phi_{G}+2(1+\beta)(1-\Phi)\right]}
$$

The density function is $\Phi_{G}=\frac{1}{\sigma \sqrt{2 \pi}} e^{-\frac{\left(\widehat{q}-e^{*}\right)^{2}}{2 \sigma^{2}}}$ and so $\tilde{\delta}$ is lower than 1 if and only if the variance of each shock is sufficiently small. Indeed, when the variance of each shock is large, it is extremely difficult to infer the behavior of each country which in turn can make tacit cooperation impossible to sustain.

The following Proposition establishes the comparative statics for the cut-off point.

Proposition 2 : If it exists, the cut-off point $\hat{q}$ is decreasing both in the length of the punishment period $T$ and in the spillover parameter $\beta$; the impact of an increase in the standard deviation $\sigma$ of each shock on $\widehat{q}$ is, however, indeterminate.

When cooperation is feasible, the admissible value of the difference between the observed level of public service in each country and the optimal level of investment is decreasing both in the spillover parameter and in the length of the punishment period. In other words, cooperation will be more easily sustained if punishment is harsher (greater $T$ ) or if public good spillovers are more important (greater $\beta$ ). Indeed, the value of the cut-off point that enforces cooperation between the two countries must balance two objectives. On the one hand, it must be high enough to provide the countries some incentives to cooperate. On the other hand, it must be sufficiently low to decrease the probability of triggering a punishment inappropriately. If $T$ or $\beta$ increases, the two countries have more incentives to cooperate and consequently the optimal trigger output must be lower to account for the possibility of negative shocks.

The impact of the variance of each shock on the cut-off point $\widehat{q}$ is, however, indeterminate. Increasing the standard deviation has indeed two conflicting effects on the cut-off value. First, it raises the risk of triggering a punishment inappropriately. This effect calls for a lower value of the cut-off point. Second, it diminishes the marginal impact of each country's effort on the resulting public output. This effect calls for a higher cut-off value in order to preserve the incentives to cooperate. The net effect is indeterminate and we cannot assess the impact of uncertainty on the degree of stringency of cut-off rules. In any case, as discussed above, the first-best level of effort 
$e^{*}$ can not be sustained as perfect public equilibrium if the distribution of each-country specific shock is too "noisy".

Finally, we can determine the optimal punishment length. Proposition 2 implies that longer punishment periods reduce both the trigger output and the probability that punishment is triggered inappropriately. Infinite punishment then maximizes the probability that the game remains in the cooperative phase, but at the risk of being stuck (in case of a bad shock) at the one-shot non-cooperative outcome forever. Therefore, in general and in contrast to repeated games with perfect monitoring, infinite punishment is not optimal. Indeed, on can observe that each country's equilibrium payoff (given by (10)) decreases in the length of the punishment period. Therefore, the optimal punishment length $\tilde{T}$ is the smallest $T$ satisfying the incentive constraint $R(G)=0$ and is finite if $\delta>\tilde{\delta}$. Indeed, this last condition implies that $R(\tilde{G})$ is strictly positive when the length of the punishment period is infinite, which implies that there exists a finite value of $T$ satisfying $R(\tilde{G}) \geq 0$. In addition, from the proof of Proposition 2 , we know that the $R(G)$ function increases in the length of non-cooperative episodes. This implies that the optimal punishment length $\tilde{T}$ is such that $R(\tilde{G})=0$ which in turn determines - with the first-order condition $R^{\prime}(\tilde{G})=0$ by definition of $\tilde{G}$ - the optimal trigger output $\tilde{q}$. This is summarized in Proposition 3.

Proposition 3: If $\delta>\tilde{\delta}$, there exists an optimal trigger equilibrium $(\tilde{q}, \tilde{T})$ such that $R(\tilde{G})=0$, where $\tilde{T}$ is positive and finite.

If countries are sufficiently patient, there exists an optimal trigger strategy equilibrium which supports the first-best level of effort $e^{*}$ of the stage game. In this equilibrium, there are cooperative phases of stochastic length and punishment phases that last $\tilde{T}$ periods, which are triggered when realized public output in one or both countries fall below the trigger output $\tilde{q}$.

\section{Imperfect Monitoring with Correlation}

In this Section, we assume the existence of a cut-off trigger strategy equilibrium and we analyze the impact of the correlation between the two country-specific shocks. Indeed, it is reasonable to believe that neighboring jurisdictions face similar socioeconomic environments and are likely to experience similar shocks. In the context of our framework, such an informational externality would thus make it possible to infer more accurately each country's effort in public investment. It is then tempting to conclude, that shock-interdependence would enhance efficiency. Interestingly, we show that a small correlation gives rise to a sort of information manipulation that can undermine the standard positive effect of correlation on the agency problem. 
Before proceeding, it might be useful to give an intuition of this result. As in the model without correlation, a tail test is the optimal statistical criterion for the countries to adopt. However, shockinterdependence brings some information which allows (with the use of observable variables) to make inference on actions with much higher precision than without shock-interdependence. Indeed, when shocks are independently distributed across countries, the best estimate of each country's effort is the observed level of public output. With correlated noise, however, the observed level of public output in one country can be compared to that in the other country to estimate more accurately each country's effort. Reversion to the non-cooperative outcome is then triggered when the estimated level of effort in one country (or both) - given the observed level of public output in the two countries - is lower than some threshold.

Suppose now that one country (let say country 2) produces the first-best level of effort but that country 1 considers the possibility of deviating from this level. If country 1 indeed decides to shirk, it leads to decrease the expected level of public output observed in country 1 . This in turn diminishes, for a certain threshold, the probability that the cut-off rule associated to country 1 is satisfied. But a low realization of the public signal in country 1 would also induce a low realization of the public signal in country 2 because of the positive correlation between country-specific shocks. Given that country 2 produces the first-best level of effort, this makes the cut-off rule associated to country 2 less stringent. This in turn increases the probability that the estimated level of effort in country 2 lies above the same threshold than the one corresponding to cut-off rule associated to country 1 . The net effect might be positive so that country 1 may have an incentive to decrease its effort.

We now present the formal analysis of the impact of the correlation between region-specific shocks on the incentives to cooperate. As before, public output $q_{j}$ in the $j$ th country is given by $q_{j}=e_{j}+\varepsilon_{j}$. We now consider that $\left(\varepsilon_{1}, \varepsilon_{2}\right)$ follows a normal law with mean equal to 0 and a variance-covariance matrix equal to

$$
\Sigma=\sigma^{2}\left(\begin{array}{cc}
1 & \rho \\
\rho & 1
\end{array}\right)
$$

where $\rho$ is the correlation coefficient.

Each country now uses the following cut-off strategy : (i) to produce the optimal level of effort $e^{*}$ in the first period and to continue to do so as long as the estimated level of effort in each country, given the observed levels of public output in both countries, is as high as $\widehat{q}$; (ii) if the estimated level of effort in one or both countries fall below $\widehat{q}$ at some period $t$, then to produce the non-cooperative level of effort $\widetilde{e}$ for $T$ periods. 
Let $\hat{e}_{j}$ be the estimated level of effort of country $j$ given the equilibrium behavior of the other country $e^{*}$ and the observed levels of public output in both countries $q_{1}$ and $q_{2}$. We have $\widehat{e}_{j}=E\left[q_{j}-\varepsilon_{j} \mid q_{1}, q_{2}, e^{*}\right]$ and the cut-off rule associated to each country is then

$$
\hat{e}_{1} \geq \hat{q} \Leftrightarrow q_{j}-E\left[\varepsilon_{j} \mid q_{1}, q_{2}, e^{*}\right] \geq \hat{q}
$$

Let $\varepsilon_{j}^{e}=q_{j}-e^{*}$ be the inference made on the shock in country $j$ when this country produces the optimal level of effort $e^{*}$. Since $q_{j}=e_{j}+\varepsilon_{j}$, we have that $\varepsilon_{j}^{e}=e_{j}-e^{*}+\varepsilon_{j}$. If country $j$ indeed produces the optimal level of effort, i.e. $e_{j}=e^{*}$, then the shock in that country is perfectly inferred from the observation of $q_{j}$. In this case, we then have $\varepsilon_{j}^{e}=\varepsilon_{j}$. Now, let us suppose that country 1 considers deviating from $e^{*}$ by producing some effort $e_{1}<e^{*}$ but that country 2 indeed produces $e^{*}$. The cut-off rule associated to country 1 is

$$
\begin{aligned}
\hat{e}_{1} \geq \hat{q} & \Leftrightarrow \quad E\left[q_{1}-\varepsilon_{1} \mid q_{1}, q_{2}, e^{*}\right] \geq \hat{q} \\
& \Leftrightarrow \quad q_{1}-E\left[\varepsilon_{1} \mid \varepsilon_{2}^{e}=q_{2}-e^{*}\right] \geq \hat{q} .
\end{aligned}
$$

where $E\left[\varepsilon_{1} \mid \varepsilon_{2}^{e}=q_{2}-e^{*}\right]=\rho \varepsilon_{2}^{e}=\rho \varepsilon_{2}$ since $e_{2}=e^{*}$. We then have

$$
\hat{e}_{1} \geq \hat{q} \Leftrightarrow \varepsilon_{1}-\rho \varepsilon_{2} \geq \hat{q}-e_{1}
$$

The cut-off rule associated to country 2 is

$$
\begin{aligned}
\hat{e}_{2} \geq \hat{q} & \Leftrightarrow \quad E\left[q_{2}-\varepsilon_{2} \mid q_{1}, q_{2}, e^{*}\right] \geq \hat{q} \\
& \Leftrightarrow \quad q_{2}-E\left[\varepsilon_{2} \mid \varepsilon_{1}^{e}=q_{1}-e^{*}\right] \geq \hat{q} .
\end{aligned}
$$

Assume that country 2 produces the first-best level of effort and that country 1 in fact decides to deviate from this level. In this case, the inference made on the shock in country 1 - under the belief that both countries act optimally - is $\varepsilon_{1}^{e}=e_{1}-e^{*}+\varepsilon_{1}$. We then have $E\left[\varepsilon_{2} \mid \varepsilon_{1}^{e}=q_{1}-e^{*}\right]=$ $\rho \varepsilon_{1}^{e}=\rho\left[e_{1}-e^{*}+\varepsilon_{1}\right]$. The cut-off rule associated to country 2 is then

$$
\hat{e}_{2} \geq \hat{q} \Leftrightarrow \varepsilon_{2}-\rho \varepsilon_{1} \geq \hat{q}-e^{*}+\rho\left(e_{1}-e^{*}\right)
$$

To characterize the probability that the game remains in the cooperative phase in the presence of shock-interdependence, let note $x=\varepsilon_{1}-\rho \varepsilon_{2}$ and $y=\varepsilon_{2}-\rho \varepsilon_{1}$. Both $x$ and $y$ follow a normal law with mean equal to 0 , variance equal to $\left(1-\rho^{2}\right) \sigma^{2}$ and covariance equal to $-\rho\left(1-\rho^{2}\right) \sigma^{2}$. Let $f(x, y)$ be the joint density, $f(x)$ and $f(y)$ the marginal densities and $f(y \mid x)$ the conditional density.

Using (18) and (20), cooperation is continued if and only if

$$
\left\{\begin{array}{l}
x \geq \hat{q}-e_{1} \\
y \geq \hat{q}-e^{*}+\rho\left(e_{1}-e^{*}\right)
\end{array} .\right.
$$


Since $x$ and $y$ are correlated, the probability that the game remains in the cooperative phase is then

$$
\mu\left(e_{1}, e^{*}\right)=\int_{\hat{q}-e_{1}}^{+\infty}\left[\int_{\hat{q}-e^{*}+\rho\left(e_{1}-e^{*}\right)}^{+\infty} f(y \mid x) d y\right] f(x) d x .
$$

As in the analysis without correlation, country 1 trades off the expected static benefit of deviation and the expected marginal cost from increasing the probability of triggering a T-period reversion to the Nash outcome. Therefore, the necessary and sufficient first-order condition for $e_{1}=e^{*}$ to be country 1 's best response to the predicted action of country 2 is still given by (12). Our purpose here is to investigate how an increase in the correlation coefficient $\rho$ affects this equilibrium condition. Note that the correlation coefficient has an impact both on the equilibrium probability $\mu\left(e^{*}, e^{*}\right)$ and on the marginal probability $\frac{\partial \mu}{\partial e_{1}}\left(e_{1}, e^{*}\right)$ of remaining in the cooperative phase. Unfortunately, we cannot obtain a general result as to the effect of the correlation coefficient on these probabilities. However, one can obtain the following interesting local result.

Lemma 2 : Increasing the correlation between the two signals of public output from 0 decreases both the marginal probability and the equilibrium probability of remaining in the cooperative phase.

Again, increasing the correlation between the two signals of public output from zero increases the prospect for information manipulation. When country 1 deviates, it becomes less likely that the cut-off rule associated to country 1 is satisfied but it also becomes more likely that the cut-off rule associated to country 2 is satisfied. This is because a bad realization of the public signal in country 1 would more likely result (with positive correlation) in a bad realization of the public signal in country 2. There is thus less chance that the estimated level of effort in country 2 falls below the cut-off point $\hat{q}$. It turns out that when the correlation coefficient is small, this last effect dominates the first effect. Hence, country 1 has an incentive to make a lower level of effort in order to increase the marginal probability of remaining in the cooperative phase. Put another way, for a given level of effort, increasing the correlation from 0 decreases this marginal probability.

In addition, increasing the correlation coefficient from 0 also decreases the equilibrium probability of maintaining cooperation. Though an increase in the correlation decreases global uncertainty it also makes the two continuation rules more contradictory. Indeed, $x=\varepsilon_{1}-\rho \varepsilon_{2}$ and $y=\varepsilon_{2}-\rho \varepsilon_{1}$ move in opposite directions as $\rho$ raises. To get an intuition of that result, assume that country 1 benefits from a positive shock while country 2 incurs a negative shock. In this case, the cut-off rule associated to country 1 is more likely to be satisfied while the reverse holds for country 2 . There are two reasons for this. First, this country incurred a negative shock. Second, given the positive correlation between country-specific shocks and the occurrence of a positive shock in country 1 , the 
estimated level of effort in country 2 is more likely to lie above the cut-off point. It turns out that, for small values of the correlation coefficient, the overall effect is negative which decreases the equilibrium probability of remaining in the cooperative phase. It is worth pointing out that this type of explanation is valid only for small values of the correlation coefficient. As shock-interdependence becomes more important, it is less likely to have a positive shock in one country and a negative shock in the other and the two continuation rules become less contradictory.

The following Proposition directly follows from Lemma 2.

Proposition 4 : Increasing the correlation between the two signals of public output from 0 reduces the prospects for maintaining cooperation as a perfect public equilibrium.

Increasing the correlation coefficient has the effect of shifting down the $R(G)$ function as depicted in Figure 2.

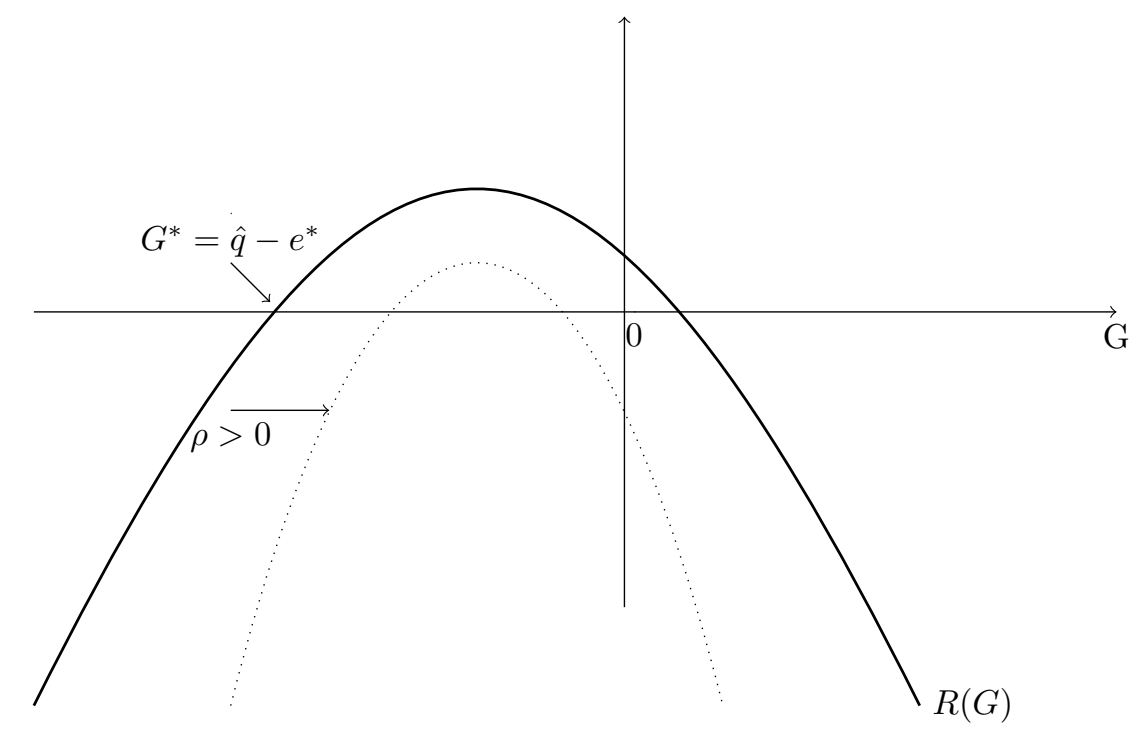

Figure 2

As shown in figure 2, the value of the cut-off point $\widehat{q}$ that prevails in the model without shockinterdependence is too low to achieve cooperation as a perfect public equilibrium when the two region-specific shocks are positively correlated. As explained above, the cross-correlation in the noisy public realization of the public good changes the inference process in a way that it decreases the marginal benefit of exerting effort. Therefore, introducing correlation between region-specific shocks requires a larger value of the cut-off point with respect to the case of independent shocks. This in turn makes cooperation more difficult to sustain in the sense that, in every period, there is more chance that the observed level of public output in one country (or both) falls below the 
cut-off point. In turn, it reduces the length of cooperative episodes as well as each country's present discounted payoff. Similarly, the optimal trigger equilibrium $(\tilde{q}, \tilde{T})$ - such that $R(\tilde{G})=0$ - corresponds to the same level of the optimal trigger output $\tilde{q}$ but to a longer length of the optimal punishment phase $\tilde{T}$, which also results in lower present discounted payoffs of cooperation.

\section{Conclusions}

We have analyzed in this paper, the possibility to sustain cooperation between two countries that make a public investment with cross-border externalities in the context of imperfect information. Even though the level of public investment provided by each country is imperfectly observed, it is shown that efficiency in local public goods provision can be sustained as a (stationary) perfect public equilibrium through a simple cut-off trigger strategy. In the absence of correlation between country-specific shocks, our comparative static results are quite intuitive. The two countries are more likely to be able to sustain cooperation if they do not discount the future too heavily or if public good spillovers are large. Introducing a marginal correlation between country-specific shocks, however, restricts the possibility of implementing intertemporal cooperation because it gives rise to a manipulation of information.

The framework analyzed in this paper might be extended on several fronts. In particular, we have focused on perfect public equilibria which use Nash reversion as punishments to support the Pareto optimal level of effort of the stage game. But this level does not necessarily corresponds to the one which maximizes the present discounted value of a cooperative arrangement in the repeated game. Furthermore, trigger strategies à la Green and Porter (1984), while subgame perfect, are not in general optimal. Abreu, Pearce and Stacchetti (1986) indeed show that optimal (symmetric) equilibria in the Green-Porter model involve punishments more severe than Nash reversion and that they have stochastic length. Another (more substantial) extension would be to characterize renegotiation-proof equilibria. Indeed, with Green and Porter's strategies but also with Abreu and al's strategies, the two countries have strong incentives to renegotiate and continue with their relationship when the public signals in one country (or both) falls and triggers punishment (especially considering that both countries did not deviate on the equilibrium path). In turn, possibility of renegotiation will (potentially) destroy countries' incentives to cooperate in the first place. ${ }^{5}$ A thorough investigation of this issue for our analysis of tacit cooperation in local public goods supply would also be interesting for future research.

\footnotetext{
${ }^{5}$ In the Green-Porter model, Chen (1995) show that the only (weakly) renegotiation-proof equilibrium is the repetition of the Cournot-Nash equilibrium if firms are restricted to playing symmetric equilibria.
} 


\section{Appendix}

\subsection{Proof of Lemma 1}

We need to check that the second order condition is satisfied i.e. $\frac{\partial^{2} V_{t}^{C}}{\partial e_{1}^{2}} \leq 0$. Consider that region 2 acts optimally i.e. $e_{2}=e^{*}$. Using (6), the second-order condition is

$$
\frac{\partial^{2} V_{t}^{C}}{\partial e_{1}^{2}}=\frac{\partial^{2} W\left(e_{1}, e^{*}\right)}{\partial e_{1}^{2}}+\delta\left[V_{t}^{C}-V_{t}^{N}\right] \frac{\partial^{2} \mu\left(e_{1}, e^{*}\right)}{\partial e_{1}^{2}} \leq 0
$$

The one-period unit of payoff for region 1 is: $W\left(e_{1}, e^{*}\right)=\frac{e_{1}+\beta e^{*}}{1+\beta}-\frac{e_{1}^{2}}{2}$. The second derivative of this function with respect to $e_{1}$ is then $\frac{\partial^{2} W\left(e_{1}, e^{*}\right)}{\partial e_{1}^{2}}=-1$. The first derivative of the probability function with respect to $e_{1}$ is given by equation (13). The second derivative of the probability function with respect to $e_{1}$ is then

$$
\frac{\partial^{2} \mu\left(e_{1}, e^{*}\right)}{\partial e_{1}^{2}}=\left(1-\Phi\left[\frac{\widehat{q}-e^{*}}{\sigma}\right]\right) \frac{\left(\widehat{q}-e_{1}\right)}{\sigma^{3} \sqrt{2 \pi}} e^{-\frac{\left(\widehat{q}-e_{1}\right)^{2}}{2 \sigma^{2}}} .
$$

The sign of this derivative is indeterminate and depends on the sign of $\left(\widehat{q}-e_{1}\right)$. Therefore, the sign of $\frac{\partial^{2} V_{t}^{C}}{\partial e_{1}^{2}}$ is also indeterminate. However, differentiating a third time, we have

$$
\frac{\partial^{3} V_{t}^{C}}{\partial e_{1}^{3}}=\frac{\partial^{3} \mu\left(e_{1}, e^{*}\right)}{\partial e_{1}^{3}}=\left(1-\Phi\left[\frac{\widehat{q}-e^{*}}{\sigma}\right]\right) \frac{\left(\widehat{q}-e_{1}\right)^{2}}{\sigma^{5} \sqrt{2 \pi}} e^{-\frac{\left(\widehat{q}-e_{1}\right)^{2}}{2 \sigma^{2}}} \geq 0 .
$$

Hence, $\frac{\partial^{2} V_{t}^{C}}{\partial e_{1}^{2}}$ is increasing in $e_{1}$ and in the limit, we have: $\lim _{e_{1} \rightarrow \infty} \frac{\partial^{2} \mu\left(e_{1}, e^{*}\right)}{\partial e_{1}^{2}}=0$ which implies that the second-order condition is always satisfied.

\subsection{Proof of Proposition 1}

To simplify the presentation, we now simply write $\Phi$ instead of $\Phi\left(\frac{G}{\sigma}\right)$. We first prove that the $R(G)$ function has a unique maximum. The derivative of $R(G)$ with respect to $G$ is given by

$$
R^{\prime}(G)=\frac{\beta^{2} \delta\left(1-\delta^{T}\right)}{2(1+\beta)^{2}}\left[\frac{U_{G}(\Phi) V(\Phi)-U(\Phi) V_{G}(\Phi)}{(V(\Phi))^{2}}\right]
$$

where $U(\Phi)=\Phi_{G}(1-\Phi), V(\Phi)=1-\delta(1-\Phi)^{2}-\delta^{T+1}\left(1-(1-\Phi)^{2}\right)$ and where $\Phi_{G}$ is the density function. Let $\Phi_{G G}$ be the derivative of $\Phi_{G}$ with respect to $G$. We have $U_{G}(\Phi)=\Phi_{G G}(1-\Phi)-\left(\Phi_{G}\right)^{2}$ and $V_{G}(\Phi)=2 \delta\left(1-\delta^{T}\right)(1-\Phi) \Phi_{G}$. Note that $\Phi_{G G}=-\frac{G}{\sigma^{2}} \Phi_{G}$ since $\Phi_{G}=\frac{1}{\sigma \sqrt{2 \pi}} e^{-\frac{G^{2}}{2 \sigma^{2}}}$. Hence $U_{G}(\Phi)=-\Phi_{G}\left[\frac{G}{\sigma^{2}}(1-\Phi)+\Phi_{G}\right]$. Inserting these expressions into $R^{\prime}(G)$ yields

$$
R^{\prime}(G)=\varphi \frac{-\Phi_{G}}{[V(\Phi)]^{2}}\left[\begin{array}{c}
{\left[1-\delta(1-\Phi)^{2}-\delta^{T+1}\left(1-(1-\Phi)^{2}\right)\right]\left(\frac{G}{\sigma^{2}}(1-\Phi)+\Phi_{G}\right)} \\
+2 \delta\left(1-\delta^{T}\right)(1-\Phi)^{2} \Phi_{G}
\end{array}\right]
$$

where $\varphi=\beta^{2} \delta\left(1-\delta^{T}\right) /\left[2(1+\beta)^{2}\right]$.

Let $\tilde{G}$ such that $R^{\prime}(\tilde{G})=0$ and denote $A(G)$ the expression in the [.]. For the FOC to be satisfied, we must have $\frac{\tilde{G}}{\sigma^{2}}(1-\Phi)+\Phi_{G}<0$ which implies that $\tilde{G}<0$. Calculating the derivative of $R^{\prime}(G)=$ $\varphi \frac{-\Phi_{G} A(G)}{[V(\Phi)]^{2}}$ with respect to $G$, one finds

$$
R^{\prime \prime}(G)=\varphi\left\{-\frac{\Phi_{G G} A(G)+\Phi_{G} A^{\prime}(G)}{[V(\Phi)]^{2}}+\frac{2 \Phi_{G} A(G) V^{\prime}(\Phi)}{[V(\Phi)]^{3}}\right\}
$$


At $G=\tilde{G}, R^{\prime}(\tilde{G})=A(\tilde{G})=0$. Therefore $R^{\prime \prime}(\tilde{G}) \leq 0$ if and only if $A^{\prime}(\tilde{G}) \geq 0$. One can show that

$$
A^{\prime}(G)=\left(\frac{(1-\Phi)}{\sigma^{2}}-\frac{2 \Phi_{G} G}{\sigma^{2}}\right)\left[1-\delta(1-\Phi)^{2}-\delta^{T+1}\left(1-(1-\Phi)^{2}\right)\right]-2 \delta\left(1-\delta^{T}\right)\left[\Phi_{G}\right]^{2}(1-\Phi)
$$

$R^{\prime}(\tilde{G})=0$ implies that

$$
2 \delta\left(1-\delta^{T}\right)(1-\Phi)\left[\Phi_{G}\right]^{2}=\left(1-\delta(1-\Phi)^{2}-\delta^{T+1}\left(1-(1-\Phi)^{2}\right)\right)\left(\frac{\Phi_{G} G}{\sigma^{2}}+\frac{\left[\Phi_{G}\right]^{2}}{(1-\Phi))}\right)
$$

Substituting this expression into $A^{\prime}(G)$ yields

$$
A^{\prime}(\tilde{G})=\left[1-\delta(1-\Phi)^{2}-\delta^{T+1}\left(1-(1-\Phi)^{2}\right)\right] \cdot\left(\frac{(1-\Phi)}{\sigma^{2}}+\frac{\left[\Phi_{G}\right]^{2}}{(1-\Phi)}-\frac{\Phi_{G} \tilde{G}}{\sigma^{2}}\right)
$$

which is strictly positive since $\tilde{G}<0$, which implies that $R^{\prime \prime}(\tilde{G}) \leq 0$. Hence, for every point that satisfies the first-order condition, the second derivative is negative i.e. $R^{\prime \prime}(\tilde{G}) \leq 0$. Therefore, the $R($.$) function$ has a unique maximum in $\tilde{G}$. (If the $R($.) function had more than one maximum, the $R($.$) function would$ have a minimum and hence a point that would satisfy the first-order condition and where the second derivative would have been positive). Consequently, a necessary and sufficient condition for the existence of a cut-off point $\widehat{q}$ such that the two countries have no incentives to deviate from the cooperative agreement is $R(\tilde{G}) \geq 0$. Indeed, if this condition is satisfied, it implies that there exists $G^{*}<\tilde{G}$ with $R\left(G^{*}\right)=0$.

\subsection{Proof of Proposition 2}

Using the implicit function theorem, we have $\frac{\partial G^{*}}{\partial T}=-\frac{\partial R\left(G^{*}\right) / \partial T}{\partial R\left(G^{*}\right) / \partial G^{*}}$. Under the condition of existence of a trigger strategy equilibrium, we have $R^{\prime}\left(G^{*}\right)>0$ since the $R\left(\right.$.) function has a unique maximum $\widetilde{G}>G^{*}$. We also have

$$
\frac{\partial R(G)}{\partial T}=-\frac{\beta^{2} \Phi_{G}(1-\Phi)}{2(1+\beta)^{2}} \frac{(1-\delta) \delta^{T+1}(\log \delta)}{\left[1-\delta(1-\Phi)^{2}-\delta^{T+1}\left(1-(1-\Phi)^{2}\right)\right]^{2}}
$$

which is positive since $\log \delta<0$. This implies that $\frac{\partial G^{*}}{\partial T}<0$.

Similarly, the change in the equilibrium value of $G^{*}$ following a change in the discount parameter $\beta$ is given by $\frac{\partial G^{*}}{\partial \beta}=-\frac{\partial R\left(G^{*}\right) / \partial \beta}{\partial R\left(G^{*}\right) / \partial G^{*}}$. Again $R^{\prime}\left(G^{*}\right)>0$ (under the condition of existence) and the derivative of $R\left(G^{*}\right)$ with respect to $\beta$ is given by

$$
\begin{aligned}
\frac{\partial R\left(G^{*}\right)}{\partial \beta} & =-\frac{1}{(1+\beta)^{2}}+\frac{2 \beta}{(1+\beta)^{3}} \frac{\delta\left(1-\delta^{T}\right) \Phi^{\prime}(1-\Phi)}{2\left[1-\delta(1-\Phi)^{2}-\delta^{T+1}\left(1-(1-\Phi)^{2}\right)\right]} \\
& =\frac{R(G)}{\beta(1+\beta)}+\frac{\beta}{(1+\beta)^{3}} \frac{\delta\left(1-\delta^{T}\right) \Phi^{\prime}(1-\Phi)}{2\left[1-\delta(1-\Phi)^{2}-\delta^{T+1}\left(1-(1-\Phi)^{2}\right)\right]}
\end{aligned}
$$

For $G=G^{*}, R\left(G^{*}\right)=0$ and so $\frac{\partial R\left(G^{*}\right)}{\partial \beta}>0$. Therefore, we have $\frac{\partial G^{*}}{\partial \beta}<0$.

Finally, the change in the equilibrium value of $G^{*}$ following a change in the variance of each specificcountry shock $\sigma$ is given by $\frac{\partial G^{*}}{\partial \sigma}=-\frac{\partial R\left(G^{*}\right) / \partial \sigma}{\partial R\left(G^{*}\right) / \partial G^{*}}$. Again, $R^{\prime}\left(G^{*}\right)>0$ and we also have

$$
\frac{\partial R\left(G^{*}\right)}{\partial \sigma}=\frac{\beta^{2} \delta\left(1-\delta^{T}\right)}{2(1+\beta)^{2}}\left[\frac{U_{\sigma}(\Phi) V(\Phi)-U(\Phi) V_{\sigma}(\Phi)}{(V(\Phi))^{2}}\right]
$$


where (again) $U(\Phi)=\Phi_{G}(1-\Phi), V(\Phi)=1-\delta(1-\Phi)^{2}-\delta^{T+1}\left(1-(1-\Phi)^{2}\right)$. Let use the following notations : $\Phi_{\sigma}=\partial \Phi\left(\frac{G}{\sigma}\right) / \partial \sigma$ and $\Phi_{G \sigma}=\partial^{2} \Phi\left(\frac{G}{\sigma}\right) / \partial G \partial \sigma=\partial \Phi_{G}\left(\frac{G}{\sigma}\right) / \partial \sigma$. We then have $U_{\sigma}(\Phi)=$ $\Phi_{G \sigma}(1-\Phi)-\Phi_{G} \Phi_{\sigma}$ and $V_{\sigma}(\Phi)=2 \delta\left(1-\delta^{T}\right)(1-\Phi) \Phi_{\sigma}$. Recall that $\Phi_{G}=\frac{1}{\sigma \sqrt{2 \pi}} e^{-\frac{\sigma^{2}}{2 \sigma^{2}}}$. Hence, $\Phi_{\sigma}=-\frac{G}{\sigma^{2} \sqrt{2 \pi}} e^{-\frac{G^{2}}{2 \sigma^{2}}}=-\frac{G}{\sigma} \Phi_{G}$ and $\Phi_{G \sigma}=\Phi_{G}\left(\frac{G^{2}}{\sigma^{3}}-\frac{1}{\sigma}\right)$. Therefore, $U_{\sigma}(\Phi)=\Phi_{G}\left[(1-\Phi)\left(\frac{G^{2}}{\sigma^{3}}-\frac{1}{\sigma}\right)+\frac{G}{\sigma} \Phi_{G}\right]$ and $V_{\sigma}(\Phi)=-\frac{G}{\sigma} 2 \delta\left(1-\delta^{T}\right)(1-\Phi) \Phi_{G}$. Inserting these expressions into $\frac{\partial R\left(G^{*}\right)}{\partial \sigma}$ yields

$$
\frac{\partial R\left(G^{*}\right)}{\partial \sigma}=\varphi \frac{\Phi_{G}}{[V(\Phi)]^{2}}\left[\begin{array}{c}
\left((1-\Phi)\left(\frac{G^{2}}{\sigma^{3}}-\frac{1}{\sigma}\right)+\frac{G}{\sigma} \Phi_{G}\right)\left[1-\delta(1-\Phi)^{2}-\delta^{T+1}\left(1-(1-\Phi)^{2}\right)\right] \\
+\frac{G}{\sigma} 2 \delta\left(1-\delta^{T}\right)(1-\Phi)^{2} \Phi_{G}
\end{array}\right]
$$

where again $\varphi=\beta^{2} \delta\left(1-\delta^{T}\right) /\left[2(1+\beta)^{2}\right]$.

We also have $R\left(G^{*}\right)=0$ which implies that

$$
\delta\left(1-\delta^{T}\right) \Phi_{G}(1-\Phi)=\frac{2(1+\beta)}{\beta}\left[1-\delta(1-\Phi)^{2}-\delta^{T+1}\left(1-(1-\phi)^{2}\right)\right]
$$

Multiplying both terms of the equality by $2(1-\Phi) \frac{G}{\sigma}$ and substituting into $\frac{\partial R\left(G^{*}\right)}{\partial \sigma}$, one can obtain

$$
\begin{aligned}
\frac{\partial R\left(G^{*}\right)}{\partial \sigma}= & \varphi \frac{\Phi_{G}}{[V(\Phi)]^{2} \sigma}\left[1-\delta(1-\Phi)^{2}-\delta^{T+1}\left(1-(1-\Phi)^{2}\right] *\right. \\
& {\left[G^{*}\left((1-\Phi) \frac{G^{*}}{\sigma^{2}}+\Phi_{G}\right)+(1-\Phi)\left(\frac{4(1+\beta) G^{*}}{\beta}-1\right)\right] }
\end{aligned}
$$

The first term in brackets is positive. The second term in brackets is the sum of two terms. The first of these terms is positive. Indeed, from the proof that the $R($.) function has a unique maximum $\tilde{G}$ which satisfies $R^{\prime}(\tilde{G})=0$, we have $(1-\Phi) \frac{G^{*}}{\sigma}+\Phi_{G^{*}}<0$ and $G^{*}<\widetilde{G}<0$. The second term of the second term in brackets is negative. The sign of $\frac{\partial R\left(G^{*}\right)}{\partial \sigma}$ is therefore indeterminate.

\subsection{Proof of Lemma 2}

Let us start by studying the impact of the correlation coefficient on the equilibrium probability given by

$$
\mu\left(e^{*}\right)=\int_{\hat{q}-e^{*}}^{+\infty}\left[\int_{\hat{q}-e^{*}}^{+\infty} f(y \mid x) d y\right] f(x) d x
$$

where

$$
f(x)=\frac{1}{\sqrt{2 \pi}} \frac{1}{\sigma \sqrt{1-\rho^{2}}} \exp ^{-\frac{x^{2}}{2 \sigma^{2}\left(1-\rho^{2}\right)}} \text { and } f(y \mid x)=\frac{1}{\sqrt{2 \pi}} \frac{1}{\sigma\left(1-\rho^{2}\right)} \exp ^{-\frac{(y+\rho x)^{2}}{2 \sigma^{2}\left(1-\rho^{2}\right)^{2}}} .
$$

Differentiating the equilibrium probability of remaining in the cooperative phase with respect to the correlation coefficient, yields

$$
\frac{\partial \mu\left(e^{*}\right)}{\partial \rho}=\int_{\hat{q}-e^{*}}^{+\infty} \frac{d f(x)}{d \rho}\left[\int_{\hat{q}-e^{*}}^{+\infty} f(y \mid x) d y\right] d x+\int_{\hat{q}-e^{*}}^{+\infty} f(x)\left[\int_{\hat{q}-e^{*}}^{+\infty} \frac{d f(y \mid x)}{d \rho} d y\right] d x .
$$

We focus on an increase in $\rho$ from 0 . It is easily verified that

$$
\left.\frac{d f(x)}{d \rho}\right|_{\rho=0}=0 \text { and }\left.\frac{d f(y \mid x)}{d \rho}\right|_{\rho=0}=-\frac{1}{\sqrt{2 \pi} \sigma} \frac{\exp ^{-\frac{y^{2}}{2 \sigma^{2}}}}{\sigma^{2}} x y .
$$


Therefore,

$$
\left.\frac{\partial \mu\left(e^{*}\right)}{\partial \rho}\right|_{\rho=0}=-\left[\frac{1}{\sigma} \int_{\hat{q}-e^{*}}^{+\infty} \frac{\exp ^{-\frac{x^{2}}{2 \sigma^{2}}}}{\sqrt{2 \pi} \sigma} x d x\right]^{2}<0 .
$$

Let us turn to the impact of the correlation coefficient on the marginal probability $\frac{\partial \mu\left(e_{1}, e^{*}\right)}{\partial e_{1}}$ of remaining in the cooperative phase. Using (23), we have that

$$
\frac{\partial \mu\left(e_{1}, e^{*}\right)}{\partial e_{1}}=\left[\int_{\hat{q}-e^{*}+\rho\left(e_{1}-e^{*}\right)}^{+\infty} f\left(y \mid \hat{q}-e_{1}\right) d y\right] f\left(\hat{q}-e_{1}\right)-\rho \int_{\hat{q}-e_{1}}^{+\infty} f\left(\hat{q}-e^{*}+\rho\left(e_{1}-e^{*}\right) \mid x\right) f(x) d x .
$$

Let evaluate this expression at $e_{1}=e^{*}$. We have

$$
\left.\frac{\partial \mu\left(e_{1}, e^{*}\right)}{\partial e_{1}}\right|_{e_{1}=e^{*}}=\left[\int_{\hat{q}-e^{*}}^{+\infty} f\left(y \mid \hat{q}-e^{*}\right) d y\right] f\left(\hat{q}-e^{*}\right)-\rho \int_{\hat{q}-e_{1}}^{+\infty} f\left(\hat{q}-e^{*} \mid x\right) f(x) d x
$$

Differentiating the above expression with respect to $\rho$ yields

$$
\begin{aligned}
\frac{\partial\left(\left.\frac{\partial \mu\left(e_{1}, e^{*}\right)}{\partial e_{1}}\right|_{e_{1}=e^{*}}\right)}{\partial \rho}= & \frac{\partial f\left(\hat{q}-e^{*}\right)}{\partial \rho} \int_{\hat{q}-e^{*}}^{+\infty} f\left(y \mid \hat{q}-e^{*}\right) d y+f\left(\hat{q}-e^{*}\right) \int_{\hat{q}-e^{*}}^{+\infty} \frac{\partial f\left(y \mid \hat{q}-e^{*}\right)}{\partial \rho} d y \\
& -\int_{\hat{q}-e^{*}}^{+\infty} f\left(\hat{q}-e^{*} \mid x\right) f(x) d x-\rho \int_{\hat{q}-e^{*}}^{+\infty} \frac{\partial f\left(\hat{q}-e^{*} \mid x\right)}{\partial \rho} f(x) d x \\
& -\rho \int_{\hat{q}-e^{*}}^{+\infty} f\left(\hat{q}-e^{*} \mid x\right) \frac{\partial f(x)}{\partial \rho} d x
\end{aligned}
$$

Now let evaluate this expression at $\rho=0$, we have

$$
\begin{aligned}
\left.\frac{\partial\left(\left.\frac{\partial \mu\left(e_{1}, e^{*}\right)}{\partial e_{1}}\right|_{e_{1}=e^{*}}\right)}{\partial \rho}\right|_{\rho=0}= & \left.\frac{\partial f\left(\hat{q}-e^{*}\right)}{\partial \rho}\right|_{\rho=0} \int_{\hat{q}-e^{*}}^{+\infty} f\left(y \mid \hat{q}-e^{*}\right) d y \\
& +\left.f\left(\hat{q}-e^{*}\right) \int_{\hat{q}-e^{*}}^{+\infty} \frac{\partial f\left(y \mid \hat{q}-e^{*}\right)}{\partial \rho}\right|_{\rho=0} d y-\int_{\hat{q}-e^{*}}^{+\infty} f\left(\hat{q}-e^{*} \mid x\right) f(x) d x
\end{aligned}
$$

Again, it is easily verified that $\left.\frac{\partial f\left(\hat{q}-e^{*}\right)}{\partial \rho}\right|_{\rho=0}=0$ and $\left.\frac{\partial f\left(y \mid \hat{q}-e^{*}\right)}{\partial \rho}\right|_{\rho=0}=-\frac{\left(\hat{q}-e^{*}\right)}{\sigma^{2}} \frac{\exp -\frac{y^{2}}{2 \sigma^{2}}}{\sqrt{2 \pi} \sigma} y$. Hence, the above expression can be rewritten as it follows

$$
\left.\frac{\partial\left(\left.\frac{\partial \mu\left(e_{1}, e^{*}\right)}{\partial e_{1}}\right|_{e_{1}=e^{*}}\right)}{\partial \rho}\right|_{\rho=0}=-\frac{\left(\hat{q}-e^{*}\right)}{\sigma^{2}} f\left(\hat{q}-e^{*}\right) \int_{\hat{q}-e^{*}}^{+\infty} \frac{\exp ^{-\frac{y^{2}}{2 \sigma^{2}}}}{\sqrt{2 \pi} \sigma} y d y-f\left(\hat{q}-e^{*}\right) \int_{\hat{q}-e^{*}}^{+\infty} \frac{\exp ^{-\frac{x^{2}}{2 \sigma^{2}}}}{\sqrt{2 \pi} \sigma} d x
$$

One can equivalently rewrite the above expression as

$$
\left.\frac{\partial\left(\left.\frac{\partial \mu\left(e_{1}, e^{*}\right)}{\partial e_{1}}\right|_{e_{1}=e^{*}}\right)}{\partial \rho}\right|_{\rho=0}=-f\left(\hat{q}-e^{*}\right)\left[\frac{\left(\hat{q}-e^{*}\right)}{\sigma} \int_{\frac{\hat{q}-e^{*}}{\sigma}}^{+\infty} \frac{\exp ^{-\frac{y^{2}}{2}}}{\sqrt{2 \pi}} y d y+\int_{\frac{\hat{q}-e^{*}}{\sigma}}^{+\infty} \frac{\exp ^{-\frac{x^{2}}{2}}}{\sqrt{2 \pi}} d x\right] .
$$

Let us study the term in brackets. Let $Z \equiv \frac{\hat{q}-e^{*}}{\sigma}$ and let define the $H($.$) function as$

$$
H(Z)=Z \int_{Z}^{+\infty} \frac{\exp ^{-\frac{t^{2}}{2}}}{\sqrt{2 \pi}} t d t+\int_{Z}^{+\infty} \frac{\exp ^{-\frac{t^{2}}{2}}}{\sqrt{2 \pi}} d t
$$

When $\rho=0$, it has been shown in the previous section that $\hat{q}<e^{*}$. Therefore, $Z$ is negative. We then study the $H($.$) function for all negative values of Z$.

Since $H^{\prime \prime}(Z)=\frac{Z \exp ^{-\frac{Z^{2}}{2}}}{\sqrt{2 \pi}}\left(Z^{2}-2\right), H^{\prime}$ is first decreasing and then increasing over $\left.]-\infty, 0\right]$. In addition, it is easily verified that $H^{\prime}(Z)=\int_{Z}^{+\infty} \frac{\exp ^{-\frac{t^{2}}{2}}}{\sqrt{2 \pi}} t d t-\left(Z^{2}+1\right) \frac{\exp ^{-\frac{Z^{2}}{2}}}{\sqrt{2 \pi}}$ is equal to 0 at the boundaries. Therefore, $H^{\prime}$ is non positive for all $Z \leq 0$ which implies that $H(Z)$ is a decreasing function. Since, $H(0)=1 / 2>0$, it follows that $H(Z) \geq 0$ for all $Z \leq 0$. We then have that $\left.\frac{\partial\left(\left.\frac{\partial \mu\left(e_{1}, e^{*}\right)}{\partial e_{1}}\right|_{\left.e_{1}=e^{*}\right)}\right.}{\partial \rho}\right|_{\rho=0}<0$. 


\subsection{Proof of Proposition 4}

The proof of this Proposition directly follows from Lemma 2. The sign of the derivative of the LHS of equation (12) with respect to $\rho$ is the same as the sign of

$$
\frac{\partial\left(\left.\frac{\partial \mu\left(e_{1}, e^{*}\right)}{\partial e_{1}}\right|_{e_{1}=e^{*}}\right)}{\partial \rho}\left[1-\delta \mu\left(e^{*}\right)-\delta^{T+1}\left(1-\mu\left(e^{*}\right)\right)\right]+\left.\frac{\partial \mu\left(e_{1}, e^{*}\right)}{\partial e_{1}}\right|_{e_{1}=e^{*}} \delta\left(1-\delta^{T}\right) \frac{\partial \mu\left(e^{*}\right)}{\partial \rho}
$$

Using lemma 2, this expression is negative for $\rho=0$ since $\left.\frac{\partial\left(\left.\frac{\partial \mu\left(e_{1}, e^{*}\right)}{\partial e_{1}}\right|_{\left.e_{1}=e^{*}\right)}\right.}{\partial \rho}\right|_{\rho=0}<0$ and $\left.\frac{\partial \mu\left(e^{*}\right)}{\partial \rho}\right|_{\rho=0}$. Therefore, increasing correlation has the effect of shifting down the $R(G)$ function and so $G^{*}$ (if it exists) raises, which amounts to making cooperation more difficult.

\section{References}

[1] Abreu, D., Pearce, D., and E. Stacchetti, (1986). Optimal Cartel Equilibria with Imperfect Monitoring, Journal of Economic Theory 39, 251-269.

[2] Abreu, D., Pearce, D., and E. Stacchetti, (1990). Toward a Theory of Discounted Repeated Games with Imperfect Monitoring, Econometrica 58, 1041-1063.

[3] Belleflamme, P., Hindriks, J., (2005). Yardstick Competition and Political Agency Problems. Social Choice and Welfare 24, 155169.

[4] Besley, T., Case, A., (1995). Incumbent Behavior: Vote-seeking, Tax setting, and Yardstick Competition. American Economic Review 85, 2545.

[5] Besley, T., Smart, M., (2007). Fiscal Restraints and Voter Welfare. Journal of Public Economics 91,755773 .

[6] Chen, K.P., (1995), On Renegotiation-Proof Equilibrium Under Imperfect Monitoring, Journal of Economic Theory 65, 600-10.

[7] Cornes, R.C., and E.C.D. Silva, (2000). Local Public Goods, Risk Sharing and Private Information in Federal Systems, Journal of Urban Economics 47, 39-60.

[8] Cornes, R.C., and E.C.D. Silva, (2002). Local Public Goods, Inter-Regional Transfers and Private Information, European Economic Review 46, 329-56.

[9] Costello, D, (1993), Fiscal Federalism in Theory and in Practice. In: The Economics of Community Public Finance, European Economy: Report and Studies Nº, Brussels.

[10] Fudenberg, D., Levine, D. and E. Maskin, (1994). The Folk Theorem with Imperfect Public Information, Econometrica 62, 997-1039.

[11] Green, E., and R. Porter, (1984). Non-cooperative Collusion under Imperfect Price Information, Econometrica 52, 87-100. 
[12] Lockwood, G., (1999). Inter-regional Insurance, Journal of Public Economics 72, 1-37.

[13] McMillan, (1979). Individual Incentives in the Supply of Public Inputs, Journal of Public Economics 12, 97-98.

[14] Mäler, K.G., (1991). Environmental Issues in the New Europe, in Atkinson, A.B. and R. Brunetta, eds., Economics for the New Europe. New York University Press, New York.

[15] Pecorino, P., (1999). The Effect of Group Size on Public Good Provision in a Repeated Game Setting, Journal of Public Economics 72, 121-134.

[16] Porter, R., (1983). Optimal Cartel Trigger Price Strategies, Journal of Economic Theory 29, 313-338.

[17] Revelli, F., (2006). Performance Rating and Yardstick Competition in Social Service Provision. Journal of Public Economics 90, 459475

[18] Salmon, P, (1987). Decentralization as an incentive scheme. Oxford Review of Economic Policy $3(2), 24-43$.

[19] Sand-Zantman, W., (2004). Economic Integration and Political Accountability, European Economic Review 48, 1001-1025. 